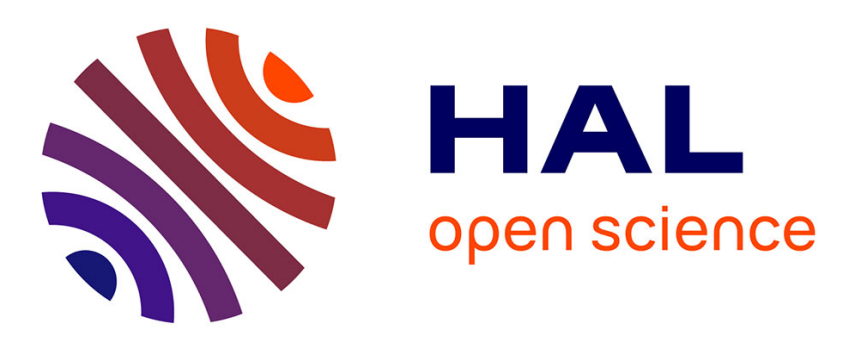

\title{
Low complexity TOA estimator for multiuser DS-UWB system
}

Hang Ma, Pascal Acco, Marie-Laure Boucheret, Danièle Fournier-Prunaret

\section{To cite this version:}

Hang Ma, Pascal Acco, Marie-Laure Boucheret, Danièle Fournier-Prunaret. Low complexity TOA estimator for multiuser DS-UWB system. 10th Workshop on Positioning Navigation and Communication (WPNC 2013), Mar 2013, Dresden, Germany. pp. 1-6. hal-01231779

\section{HAL Id: hal-01231779 \\ https://hal.science/hal-01231779}

Submitted on 20 Nov 2015

HAL is a multi-disciplinary open access archive for the deposit and dissemination of scientific research documents, whether they are published or not. The documents may come from teaching and research institutions in France or abroad, or from public or private research centers.
L'archive ouverte pluridisciplinaire HAL, est destinée au dépôt et à la diffusion de documents scientifiques de niveau recherche, publiés ou non, émanant des établissements d'enseignement et de recherche français ou étrangers, des laboratoires publics ou privés. 


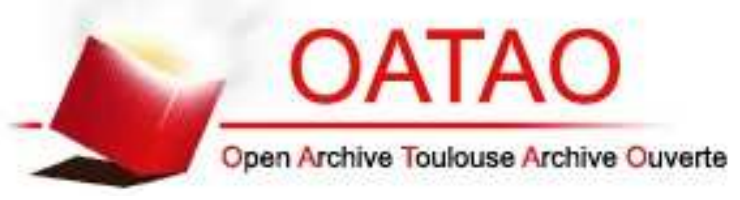

\section{Open Archive TOULOUSE Archive Ouverte (OATAO)}

OATAO is an open access repository that collects the work of Toulouse researchers and makes it freely available over the web where possible.

This is an author-deposited version published in : http://oatao.univ-toulouse.fr/ Eprints ID : 12575

The contribution was presented at WPNC 2013 :

http://wpnc.net/wpnc13.html

Official URL: http://dx.doi.org/10.1109/WPNC.2013.6533284

To cite this version : Ma, Hang and Acco, Pascal and Boucheret, Marie-Laure and Fournier-Prunaret, Daniele Low complexity TOA estimator for multiuser DS-UWB system. (2013) In: 10th Workshop on Positioning Navigation and Communication (WPNC 2013), 20 March 2013 - 21 March 2013 (Dresden, Germany).

Any correspondance concerning this service should be sent to the repository administrator: staff-oatao@listes-diff.inp-toulouse.fr 


\title{
Low Complexity TOA Estimator for Multiuser DS-UWB System
}

\author{
Hang $\mathrm{Ma}^{*}$, Pascal Acco* , Marie-Laure Boucheret ${ }^{\dagger}$ and Daniele Fournier-Prunaret* \\ ${ }^{*}$ CNRS-LAAS, INSA \\ Univ de Toulouse, Toulouse, France \\ Email: \{hma, pascal.acco, daniele.fournier\}@insa-toulouse.fr \\ ${ }^{\dagger}$ IRIT/INP-ENSEEIHT \\ University of Toulouse, Toulouse, France \\ Email: marie-laure.boucheret@enseeiht.fr
}

\begin{abstract}
In this paper, we present a low complexity Time Of Arrival (TOA) estimator for direct-sequence ultra-wideband (DS-UWB) ranging system. With the assumption that TOA is the integer multiples of chip duration, our decoupled multiuser ranging (DEMR) estimator employs integrate-and-dump filter (IDF) in chip sampling rate instead of matched filter (MF) as the front-end to reduce sampling rate and to simplify the structure of estimator. This subsampling estimator is simplified substantially in dense multipath environment furthermore due to the long repetition time of DS-UWB pulse. Simulation results show that compared with other low complexity TOA estimator, DEMR estimator is not only quite near-far resistant, but also can obtain noticeable ranging performance in the fully loaded system.
\end{abstract}

Keywords-multipath channel, multiuser interference, time of arrival (TOA) estimation, Ultra-wideband (UWB).

\section{INTRODUCTION}

Ultra-wideband (UWB) signals are characterized by their extremely wide bandwidth. Due to larger bandwidth inducing high timing resolution, UWB technology offers the potential of achieving high ranging accuracy through signal Time Of Arrival (TOA) measurement [1] [2].

Various TOA estimators have been proposed in the literature [3]-[10]. In general, they can be classified into matched filter (MF)-based coherent algorithms [3]-[7], and energy detection(ED)-based non-coherent algorithms [8]-[10]. A maximum likelihood (ML) approach is proposed in [3] [4], but the computational complexity limits its implementation. Although several suboptimal TOA estimators have reduced complexity considerably [5]-[7], the requirement of Nyquist sampling rate or higher still can be impractical due to the large bandwidth of UWB signals. It is well known that MF coincides with the optimal maximum likelihood (ML) method for a single user in the presence of white Gaussian noise but its performance degrades drastically in a near-far multiuser environment. To alleviate the effects of multiuser interference (MUI), [6] designed a specific training sequence for synchronization with dirty templates. [11] proposed chiplevel blind and data-aided synchronization algorithms by using a posterior probability of each chip to suppress MUI. Although coherent algorithms can mitigate MUI, the requirement of high sampling rate is still existed.

As an alternative, subsampling TOA estimators based on energy detection (ED) have received significant attention [8][10]. While these ED based estimators are with low complexity at the expense of relatively low ranging accuracy. Meanwhile, not accounting for MUI with ED estimator can greatly degrade performance further [12]. [13] proposed a nonlinear filtering technology on received signals energy to mitigate MUI for ED estimator. [14] proposed a TOA estimation scheme to mitigate both narrowband and wideband interferences in multipath channel for ED estimator.

Moreover, [6] [11] [13] [14] are proposed for small number of users. In the case of heavy or fully loaded system, especially with the near-far effect, all these algorithms cannot achieve acceptable performance.

[15] introduced an interesting synchronization algorithm for direct sequence code-division multiple-access (DS-CDMA) system, which is referred as decoupled multiuser acquisition (DEMA) algorithm. DEMA algorithm is MF-based asymptotic ML algorithm, it is quite near-far resistant and can support large number of users. However, DEMA algorithm requires not only high sampling rate, but the computational complexity is also quite high especially in multipath environment due to a search over a multi-dimensional parameter space.

In this paper, we present a low complexity decoupled multiuser ranging (DEMR) estimator for TOA estimation in direct-sequence ultra-wideband (DS-UWB) ranging system. DEMR estimator extends DEMA algorithm [15] into DSUWB ranging system. With the assumption that TOA is the integer multiples of chip duration, we replace the MF in [15] by an integrate-and-dump filter (IDF) in chip sampling rate to reduce the sampling rate and to simplify the estimator structure. Moreover, comparing with the work of [15], this subsampling TOA estimator is simplified substantially in multipath environment due to the long repetition time of DSUWB pulse. Searching over a multi-dimensional parameter space problem is simplified to a set of one-dimensional (1D) problems. Although reducing complexity considerably, we show that DEMR estimator is quite near-far resistant and can obtain noticeable performance in fully-loaded system in the dense multipath channel.

The paper is organized as follows. In section II, our system model is introduced. In section III, we describe our TOA estimation algorithm. Numerical evaluations of the algorithm are illustrated in section IV. Finally, conclusions are given in 
section V.

\section{SySTEM MOdEL}

The system under investigation is $K$-user DS-UWB system using binary phase-shift keying (BPSK) modulation. The transmitted signal by the $k$ th user can be formed as

$$
\begin{aligned}
s_{k}(t) & =\sqrt{P_{k}} \\
& \sum_{j=-\infty}^{+\infty} \sum_{m=0}^{M-1} d_{k}(m) \sum_{n=0}^{N-1} c_{k}^{\prime}(n) p\left(t-n T_{f}-m T_{b}\right)
\end{aligned}
$$

where $P_{k}$ is the $k$ th user's transmitted power, $M$ is number of data bits considered for TOA estimation, $d_{k}(m) \in\{ \pm 1\}$ is the $m$ th transmitted bit, $c_{k}^{\prime}(n) \in\{ \pm 1\}$ is the direct spreading sequence with length $N$ of user $k . p(t)$ is the pulse shape with pulse duration $T_{p}, T_{c}$ is chip duration $T_{c} \geq T_{p} . T_{f}=N_{c} T_{c}$ is the frame duration, where $N_{c}$ is the number of chips in one frame. $T_{b}=N T_{f}$ is data bit interval. Hence, $s_{k}(t)$ can be rewritten as

$$
\begin{aligned}
s_{k}(t) & =\sqrt{P_{k}} \\
& \sum_{j=-\infty}^{+\infty} \sum_{m=0}^{M-1} d_{k}(m) \sum_{i=0}^{N N_{c}-1} g_{k}(i) p\left(t-i T_{c}-m T_{b}\right)
\end{aligned}
$$

in which $g_{k}(i) \in\{ \pm 1,0\}, g_{k}(i)$ is formed by inserted $N_{c}-1$ " 0 " between each element in $c_{k}(n)$, i.e,

$$
g_{k}(i)=\left\{\begin{array}{cc}
c_{k}^{\prime}\left(i / N_{c}\right) & \text { if } i \bmod N_{c}=0 \\
0 & \text { otherwise. }
\end{array}\right.
$$

For the case of multipath channel described in the IEEE 802.15.4a channel model [16], the received signal can be written as

$$
r(t)=\sum_{k=1}^{K} \sum_{q=1}^{L_{k}} a_{k, q} s_{k}\left(t-\tau_{k, q}\right)+n(t)
$$

where $a_{k, q}$ and $\tau_{k, q}$ denote the complex channel coefficient and time delays of the $q$ th multipath component of the $k$ th user respectively. $L_{k}$ is the number of multipath for the $k$ th user. $\tau_{k, 1}$ is the parameter of interest in TOA estimation. $n(t)$ is the additive white Gaussian noise with zero mean and double sided power spectral density of $N_{0} / 2$. For simplicity, we only consider the reception of $M$ bits, and assume first user is user of interest. Therefore,

$$
\begin{aligned}
r(t)= & \sum_{k=1}^{K} \sum_{q=1}^{L_{k}} \sqrt{P_{k}} a_{k, q} \sum_{m=0}^{M-1} d_{k}(m) \\
& \sum_{i=0}^{N N_{c}-1} g_{k}(i) p\left(t-i T_{c}-m T_{b}-\tau_{k, q}\right)+n(t) .
\end{aligned}
$$

In typical UWB ranging scenarios, with proper selection of $T_{c}$ and $N, \tau_{k, q}$ can be assumed to be bounded by a bit interval. We further assume $\tau_{k, q}$ is integer multiples of chip duration $T_{c}$. Since in UWB system, $T_{c}$ is in the order of nano-second, it is a mild assumption for ranging accuracy. Hence, the delay $\tau_{k, q}=p_{k, q} T_{c}, p_{k, q}$ is an integer within $\left\{0,1, \ldots, N N_{c}-1\right\}$. TOA estimation is equivalent to estimating $p_{k, q}$.
The receiver front-end consists of an integrate-and-dump filter (IDF) with integration time $T_{c}$. Due to the integer TOA assumption, $\mathrm{MF}$ as the receiver front-end only requires the chip sampling rate rather than Nyquist sampling rate or higher. Replacing MF by IDF reduces the computational complexity of TOA estimator further although with the cost of the loss of SNR.

Without loss of generality, we use the real-valued TOA in the simulation in the section IV. Under this case, as shown in the section IV, IDF-based DEMR estimator performs much better than MF-based DEMR estimator in chip sampling rate even with the loss of SNR.

The received sequence $\{r(l)\}$ can be expressed as

$$
\begin{gathered}
r(l)=\sum_{k=1}^{K} \sum_{q=1}^{L_{k}} \frac{1}{T_{c}} \int_{(l-1) T_{c}}^{l T_{c}} a_{k, q} s_{k}\left(t-\tau_{k, q}\right)+n(l) \\
l=1,2, \ldots, N N_{c}
\end{gathered}
$$

$n(l)$ denotes the zero-mean white Gaussian noise with variances $\sigma_{n}^{2}$. The received vector of the $m$ th bit interval is

$$
\begin{aligned}
& \mathbf{r}(m)= \\
& {\left[r\left(m N N_{c}+1\right), r\left(m N N_{c}+2\right), \ldots, r\left(m N N_{c}+N N_{c}\right)\right]^{T}}
\end{aligned}
$$

where $(\cdot)^{T}$ denotes the transpose, and $\mathbf{n}(m)$ is similarly formed by $n(l)$. The vector of sequence is defined as

$$
\mathbf{c}_{\mathbf{k}}=\left[c_{k}(1), c_{k}(2), \ldots, c_{k}\left(N N_{c}\right)\right]^{T}
$$

in which $c_{k}(l)=\frac{1}{T_{c}} \int_{(l-1) T_{c}}^{l T_{c}} \sum_{i=0}^{N N_{c}-1} g_{k}(i) p\left(t-i T_{c}\right) d t$.

In the $m$ th bit interval, due to the $\tau_{k, q}$, received signal includes $\mathbf{a}_{k}^{1}\left(\tau_{k, q}\right)$, the end part of $(m-1)$ th bit; and $\mathbf{a}_{k}^{2}\left(\tau_{k, q}\right)$, the beginning part of $m$ th bit.

$$
\begin{aligned}
& \mathbf{a}_{k}^{1}\left(\tau_{k, q}\right)=\mathbf{P}_{1}\left(p_{k, q}\right) \mathbf{c}_{k} \\
& \mathbf{a}_{k}^{2}\left(\tau_{k, q}\right)=\mathbf{P}_{2}\left(p_{k, q}\right) \mathbf{c}_{k}
\end{aligned}
$$

where $\mathbf{P}_{1}(p)$ and $\mathbf{P}_{2}(p)$ denote the $N N_{c} \times N N_{c}$ shifting matrices

$$
\mathbf{P}_{1}(p)=\left[\begin{array}{cc}
\mathbf{0} & \mathbf{I}_{p} \\
\mathbf{0} & \mathbf{0}
\end{array}\right] \quad \mathbf{P}_{2}(p)=\left[\begin{array}{cc}
\mathbf{0} & \mathbf{0} \\
\mathbf{I}_{N N_{c}-p} & \mathbf{0}
\end{array}\right]
$$

and $\mathbf{I}_{p}$ is $p \times p$ identity matrix. Then the received vector can be rewritten as

$$
\begin{gathered}
\mathbf{r}(m)=\sum_{k=1}^{K} \sum_{q=1}^{L_{k}} \beta_{k, q} \mathbf{A}_{k}\left(\tau_{k, q}\right) \mathbf{z}_{k}(m)+\mathbf{n}(m) \\
m=0,1, \ldots, M-1
\end{gathered}
$$

where

$$
\begin{gathered}
\beta_{k, q}=a_{k, q} \sqrt{P_{k}} \\
\mathbf{A}_{k}\left(\tau_{k, q}\right)=\left[\begin{array}{ll}
\mathbf{a}_{k}^{1}\left(\tau_{k, q}\right) & \mathbf{a}_{k}^{2}\left(\tau_{k, q}\right)
\end{array}\right] \\
\mathbf{z}_{k}(m)=\left[\begin{array}{ll}
d_{k}(m-1) & d_{k}(m)
\end{array}\right]^{T} .
\end{gathered}
$$

Note that when $m=0, d_{k}(-1)$ is unknown. We can choose $d_{k}(-1)=0$ which has little effect on the estimation. We rewrite $\mathbf{r}(m)$ as

$$
\mathbf{r}(m)=\mathbf{B} \mathbf{s}(m)+\mathbf{n}(m)
$$


where

$$
\begin{aligned}
& \mathbf{B}= \\
& {\left[\sum_{q=1}^{L_{1}} \beta_{1, q} \mathbf{A}_{1}\left(\tau_{1, q}\right) \sum_{q=1}^{L_{2}} \beta_{2, q} \mathbf{A}_{2}\left(\tau_{2, q}\right) \ldots \sum_{q=1}^{L_{k}} \beta_{K, q} \mathbf{A}_{k}\left(\tau_{K, q}\right)\right]} \\
& \quad \mathbf{s}(m)=\left[\mathbf{z}_{1}^{T}(m) \mathbf{z}_{2}^{T}(m) \ldots \mathbf{z}_{K}^{T}(m)\right]^{T} .
\end{aligned}
$$

Specially, we assume that user number, all users spreading sequence and data bits are known. If we let

$$
\mathbf{R}_{\mathbf{s s}}(M)=\frac{1}{M} \sum_{m=0}^{M-1} \mathbf{s}(m) \mathbf{s}^{T}(m)
$$

and assume data bits for all users are i.i.d, hence, $\mathbf{R}_{\mathrm{ss}}(M)=$ $\mathbf{I}_{2 K}$, when $M \rightarrow+\infty$ [15]. We also assume that $\mathbf{s}(m)$ and $\mathbf{n}(m)$ are uncorrelated, i.e,

$$
\lim _{M \rightarrow \infty} \frac{1}{M} \sum_{m=0}^{M-1} \mathbf{s}(m) \mathbf{n}^{H}(m)=\mathbf{0 .} .
$$

\section{Multiuser tOA Estimation}

With the received signal shown in Section II, the Maximum Likelihood (ML) estimation is equivalent to

$$
\begin{aligned}
& \left\{\left\{\hat{\beta}_{k, q}, \hat{\tau}_{k, q}\right\}_{q=1}^{L_{k}}\right\}_{k=1}^{K}=\underset{\left\{\left\{\beta_{k, q}, \tau_{k, q}\right\}_{q=1}^{L_{k}}\right\}_{k=1}^{K}}{\arg \min } \operatorname{tr}\left\{\frac{1}{M} \sum_{m=0}^{M-1}\right. \\
& \left.\left[\mathbf{r}(m)-\mathbf{B}\left(\beta_{k, q}, \tau_{k, q}\right) \mathbf{s}(m)\right]\left[\mathbf{r}(m)-\mathbf{B}\left(\beta_{k, q}, \tau_{k, q}\right) \mathbf{s}(m)\right]^{H}\right\}
\end{aligned}
$$

where $\operatorname{tr}\{\cdot\}$ is trace operator. Minimizing (21) with respect to $\mathbf{B}$ gives an unstructured estimation $\hat{\mathbf{B}}$ [15]:

$$
\begin{gathered}
\hat{\mathbf{B}}=\mathbf{R}_{\mathbf{s r}}^{H}(M) \mathbf{R}_{\mathbf{s s}}^{-\mathbf{1}}(M) \\
\mathbf{R}_{\mathbf{s r}}(M)=\frac{1}{M} \sum_{m=0}^{M-1} \mathbf{s}(m) \mathbf{r}^{H}(m) .
\end{gathered}
$$

As $\mathbf{s}(m)$ and $\mathbf{n}(m)$ are uncorrelated, if $\mathbf{R}_{\mathbf{s s}}^{-\mathbf{1}}(M)$ exists, we can find that $\hat{\mathbf{B}}$ is a $(1 \sqrt{M})$-consistent estimate of $\mathbf{B}$. Since for large $M, \mathbf{R}_{\mathbf{s s}}(M)=\mathbf{I}_{2 K}$, (21) can be decoupled into a series of $K$ minimization problems [15]. Let

$$
\hat{\mathbf{B}}=\left[\begin{array}{llll}
\hat{\mathbf{B}}_{1} & \hat{\mathbf{B}}_{2} & \ldots & \hat{\mathbf{B}}_{K}
\end{array}\right],
$$

for each user (21) is equivalent to

$$
\begin{gathered}
\left\{\hat{\beta}_{k, q}\right\}_{q=1}^{L_{k}},\left\{\hat{\tau}_{k, q}\right\}_{q=1}^{L_{k}}= \\
\underset{\substack{\arg \min \\
\left\{\beta_{k, q}\right\}_{q=1}^{L_{k}},\left\{\tau_{k, q}\right\}_{q=1}^{L_{k}}}}{k=1,2, \ldots, K} \\
k=\sum_{q=1}^{L_{k}} \beta_{k, q} \mathbf{A}_{k}\left(\tau_{k, q}\right)-\hat{\mathbf{B}}_{k} \|_{F}^{2}
\end{gathered}
$$

where $\|\cdot\|_{F}$ is Frobenius norm [15].

From (25), we can find DEMR algorithm can decouple between different users, but not between different path. Hence $3 L_{k}$-dimensional search over the parameter space (note that $\beta_{k, q}$ is complex-valued) is employed to estimate $\left\{\tau_{k, q}, \beta_{k, q}\right\}_{q=1}^{L_{k}}$ in (25). But due to the DS-UWB signal structure and integer delay assumption, the $3 L_{k}$-dimensional search problem in (25) can be simplified to the $L_{k}$ maximums in one dimensional search problem.

Let

$$
\begin{aligned}
\mathbf{a}_{k}\left(\tau_{k, q}\right) & =\operatorname{vec}\left[\mathbf{A}_{k}\left(\tau_{k, q}\right)\right] \\
\hat{\mathbf{b}}_{k} & =\operatorname{vec}\left[\hat{\mathbf{B}}_{k}\right]
\end{aligned}
$$

where $v e c[\cdot]$ denotes stacking the columns of a matrix on top of one another. We get

$$
\mathbf{a}_{k}\left(\tau_{k, q}\right)=\left[\begin{array}{l}
\mathbf{P}_{1}\left(p_{k, q}\right) \mathbf{c}_{k} \\
\mathbf{P}_{2}\left(p_{k, q}\right) \mathbf{c}_{k}
\end{array}\right],
$$

with the integer delay assumption, $\mathbf{a}_{k}\left(\tau_{k, q}\right)$ is cyclic shift of $\mathbf{a}_{k}(0)$. As $g_{k}$ is formed by inserted $N_{c}-1$ " 0 " between each element in $c_{k}^{\prime}(n)$, the stacked code $\mathbf{a}_{k}(0)$ also has "0" inserted autocorrelation function. If the multipath delay spread is much smaller than $N N_{c} T_{c}$, so $\max \left(\tau_{k, q}\right)<<N N_{c} T_{c}$, we can ignore $\mathbf{a}_{k}^{T}\left(\tau_{k, s}\right) \mathbf{a}_{k}\left(\tau_{k, r}\right)$ when $s \neq r, s, r \in\left\{1,2, \ldots, L_{k}\right\}$. Hence, stacked code $\mathbf{a}_{k}(0)$ has white noise like autocorrelation function.

With this property, minimizing the cost function (25) with respect to $\tau_{k, q}$ and $\beta_{k, q}$ yields

$$
\begin{aligned}
\left\{\hat{\tau}_{k, q}\right\}_{q=1}^{L_{k}} & =\underset{\left\{\tau_{k, q}\right\}_{q=1}^{L_{k}}}{\arg \max } \sum_{q=1}^{L_{k}} \frac{\left|\mathbf{a}_{k}^{T}\left(\tau_{k, q}\right) \hat{\mathbf{b}}_{k}\right|^{2}}{\mathbf{a}_{k}^{T}\left(\tau_{k, q}\right) \mathbf{a}_{k}\left(\tau_{k, q}\right)} \\
\left\{\hat{\beta}_{k, q}\right\}_{q=1}^{L_{k}} & =\frac{\mathbf{a}_{k}^{T}\left(\left\{\hat{\tau}_{k, q}\right\}_{q=1}^{L_{k}}\right) \hat{\mathbf{b}}_{k}}{\mathbf{a}_{k}^{T}\left(\left\{\hat{\tau}_{k, q}\right\}_{q=1}^{L_{k}}\right) \mathbf{a}_{k}\left(\left\{\hat{\tau}_{k, q}\right\}_{q=1}^{L_{k}}\right)} .
\end{aligned}
$$

Since $\mathbf{a}_{k}^{T}\left(\tau_{k, q}\right) \mathbf{a}_{k}\left(\tau_{k, q}\right)=\mathbf{a}_{k}^{T}(0) \mathbf{a}_{k}(0)$ and $\left|\mathbf{a}_{k}^{T}\left(\tau_{k, q}\right) \hat{\mathbf{b}}_{k}\right|^{2}$ is non-negative, the $L_{k}$-dimensional search problem in (29) can be simplified to $L_{k}$ independent maximum search in one dimensional with the constraint $\hat{\tau}_{k, s} \neq \hat{\tau}_{k, r}, s, r \in$ $\left\{1,2, \ldots, L_{k}\right\}$.

The maximum of (29) is simple to perform, we first rewrite (29) as

$$
\begin{gathered}
\left\{\hat{\tau}_{k, q}\right\}_{q=1}^{L_{k}}=\underset{\left\{\tau_{k, q}\right\}_{q=1}^{L_{k}}}{\arg \max } \frac{\sum_{q=1}^{L_{k}}\left|\mathbf{a}_{k}^{T}\left(\tau_{k, q}\right) \hat{\mathbf{b}}_{k}\right|^{2}}{\mathbf{a}_{k}^{T}(0) \mathbf{a}_{k}(0)} \\
R\left(\tau_{k, q}\right)=\frac{\left|\mathbf{a}_{k}^{T}\left(\tau_{k, q}\right) \hat{\mathbf{b}}_{k}\right|^{2}}{\mathbf{a}_{k}^{T}(0) \mathbf{a}_{k}(0)}
\end{gathered}
$$

$\left\{\hat{\tau}_{k, q}\right\}_{q=1}^{L_{k}}$ which correspond to the $L_{k}$-largest values of $R\left(\tau_{k, q}\right)$ are the estimations of time delays. The earliest time delay among $\left\{\hat{\tau}_{k, q}\right\}_{q=1}^{L_{k}}$ is the TOA estimation of user $k$.

In the real environment, since the exact number of multipath components $L_{k}$ is unknown, we need to use some estimation strategies to detect the TOA. X-max criterion proposed in [9] is based on the selection of the earliest $\hat{\tau}_{k, q}$ as the TOA estimation of user $k$ among $\left\{\hat{\tau}_{k, q}\right\}_{q=1}^{X}$ which corresponds to the $\mathrm{X}$ largest values of $R\left(\tau_{k, q}\right)$. Simple thresholding criterion in [2] is based on comparing the values of $R\left(\tau_{k, q}\right)$ to a 


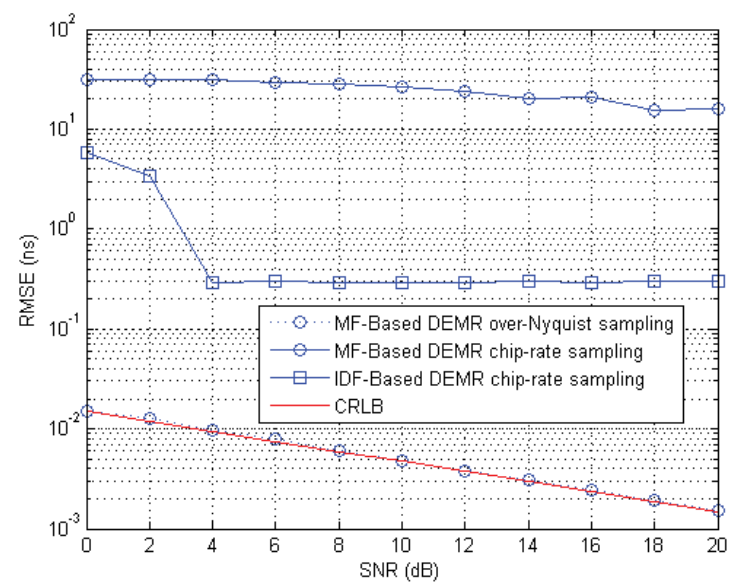

Fig. 1. RMSE versus SNR with $M=100, N=31, K=1$ in AWGN channel.

threshold, the first threshold crossing event is taken as the TOA estimation of user $k$. Recently, a new approach based on information theoretic criterion is proposed in [17] [18], this new blind estimation has no requirement of channel information or predefined threshold. In this paper, we employ the X-max criterion to detect the first path due to its comparative low complexity.

Note that DEMR algorithm cannot perform a TOA estimate until $M \geq 2 K$ for the existence of $\mathbf{R}_{\mathrm{ss}}^{-1}(M)$ [15].

\section{NUMERICAL EVALUATIONS}

In this section, we present the simulation results of the proposed algorithm. Simulations are carried out on AWGN channel and IEEE 802.15.4a channel mode 1 (CM1) which is a typical dense multipath channel for UWB systems. Performance is presented by root mean square error (RMSE) of TOA estimation.

Each user is assigned a Gold sequence of $N=31$, in the following, TOA of first user is evaluated whose transmitted power $P_{1}=1$ with no loss of generality. All interfering users were given a random received power with a log-normal distribution with a mean $d \mathrm{~dB}$ above the desired signal and a standard deviation of $10 \mathrm{~dB}$. That is $P_{k}=10^{\varepsilon_{k} / 10}$, where $\varepsilon_{k} \sim N(d, 100)$. The near-far ratio is defined as the ratio of the mean of the random powers of the interfering users to that of the desired user. Hence, the near-far radio is $d$ in decibels. SNR is defined to be $E_{b} / N_{0}$, where $E_{b}$ is energy per bit for the first user. The pulse $p(t)$ is raised cosine pulse with rolloff factor $\beta=0.6$, pulse and chip duration are set equal to 1ns, i.e., $T_{c}=T_{p}=1 n s$, and $N_{c}=16$. Time delays of channel are uniformly distributed over $[0,100 n s)$, which are the real-valued delays including the fractional part. In CM1 channel, $\mathrm{X}$ is chosen to be 3 which is 1 in AWGN channel. The results below are based on 500 Monte-Carlo trials. In each trial, different user passes through different CM1 channel realization.

As discussed in section II, DEMR can perform with both $\mathrm{MF}$ and IDF as the receiver front-end. Fig.1 depicts the ranging performance of MF-based and IDF-based DEMR estimators

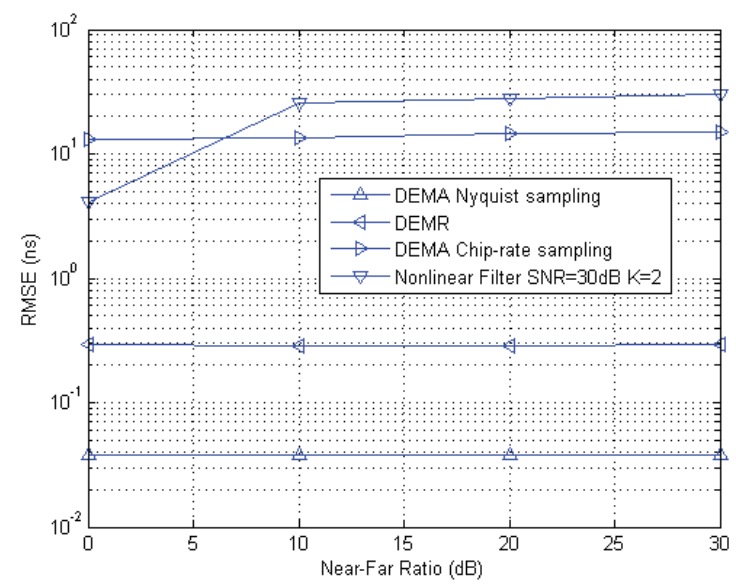

Fig. 2. RMSE versus near-far ratio for different estimators with $M=100$, $N=31, S N R=20 d B, K=10$ in AWGN channel

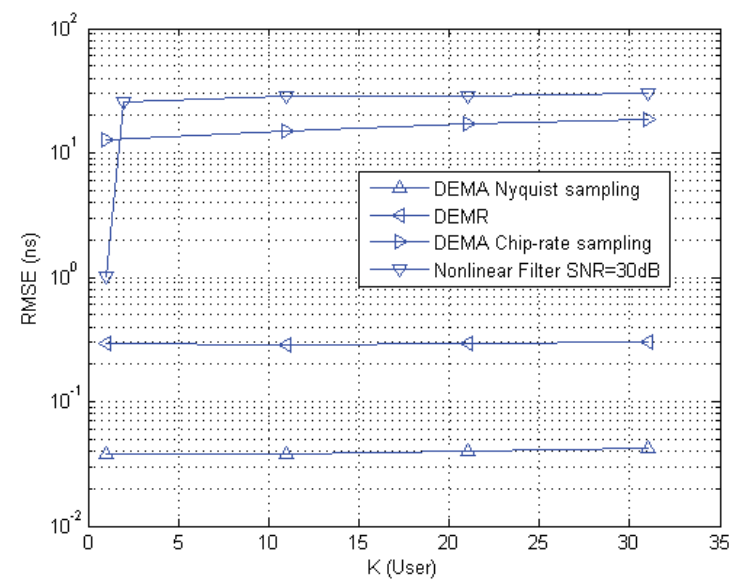

Fig. 3. RMSE versus $K$ for different estimators with $M=100, N=31$, $S N R=20 d B$ and near-far ratio is $10 \mathrm{~dB}$ in AWGN channel.

with single user in AWGN channel. In addition, CRLB are presented. Note that because of different definition of SNR, there is a $\log _{10}(N) \mathrm{dB}$ shift of CRLB compared with that in [2]. As shown in the Fig.1, although MF-based DEMR estimator can reach CRLB with over-Nyquist sampling rate, it performs much worse than IDF-based DEMR estimator in the chip sampling rate since real-valued delay is used in simulation. In medium and high SNR region, the IDF-based DEMR estimator exhibits a floor equal to $T_{c} / \sqrt{12} \approx 0.2887 \mathrm{~ns}$ since we have estimated the exact the integer part of TOA $p_{k, q}$ but ignore the fractional part of TOA. Though chip sampling rate limits the accuracy of IDF-based DEMR estimator into $T_{c} / \sqrt{12}$, its low sampling rate and complexity make it be more practical than MF-based DEMR estimator. Without particularly indicated, DEMR estimator in the following part works in chip sampling rate with IDF as front-end filter.

In Fig. 2 and Fig.3, we compare the ranging performance of DEMR estimator with 2 other estimators, namely DEMA and Nonlinear filter estimator. Nonlinear filter estimator in [13] proposed as the low complexity TOA estimator working 


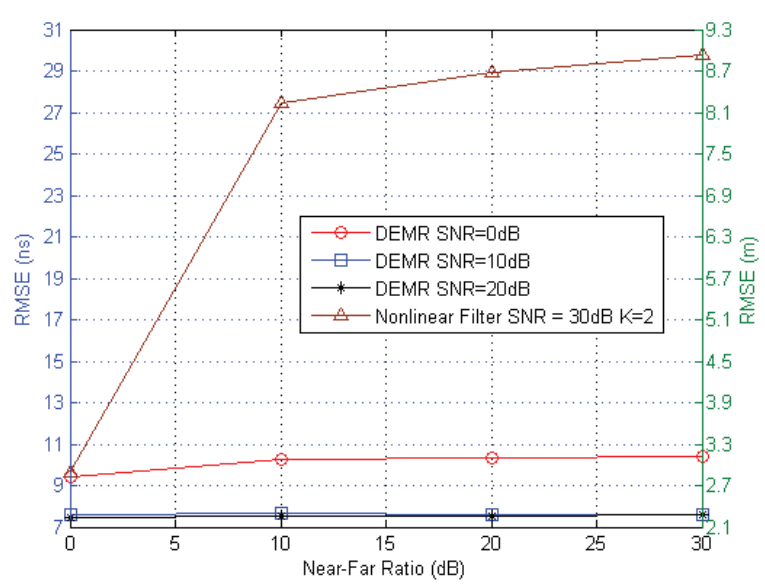

Fig. 4. RMSE versus near-far ratio for different SNR values with $M=100$, $N=31$, and $K=10$ in CM1 channel.

in sub-Nyquist sampling rate. It performs nonlinear filtering on the received signal energy to mitigate MUI for ED-based estimator. In our simulation, Nonlinear filter estimator works in chip sampling rate. [13] proposed 2 different nonlinear filters, we employ minimum filer in our simulation since it outperforms the median filter in the presence of severe MUI. Nonlinear filter estimator for DS-UWB system needs a extra burst modulation. In burst modulation, a symbol interval is equal to two data bit duration, each half of symbol interval is called burst. $s_{k}(t)$ is transmitted either in the first or the second half in a pseudorandom pattern depending on the data bit. DEMA estimator in [15] is MF-based asymptotic ML algorithm. Since we transmit the band-limited pulse, the nonlinear optimization is employed to estimate fractional delay in DEMA estimator. In Fig.2, DEMA estimator is performed in both chip-rate sampling and Nyquist sampling rate which is equal to 8 times chip sampling rate in our simulation.

RMSE as the function of near-far ratio in AWGN channel with 10 users ( 2 users for Nonlinear filter estimator) is shown in Fig.2. We can find that DEMA estimator obtains the very precious TOA estimation with Nyquist sampling rate, RMSE of which approaches to $0.0380 \mathrm{~ns}$. However, performance degrades sharply to about $13 \mathrm{~ns}$ in the chip sampling rate even with smallest near-far ratio. This severe degradation is brought by using the MF-based estimator in sub-Nyquist sampling rate. By contrast, DEMR estimator with IDF as front-end reaches noticeable ranging performance in the low chip sampling rate and almost has no influence by near-far ratio. RMSE of DEMR estimator approaches to $0.2887 \mathrm{~ns}$ which is the theoretical optimal value. As shown in the figure, Nonlinear filter estimator which also works in chip sampling rate has no robustness to near-far ratio. When near-far ratio is up to $10 \mathrm{~dB}$, RMSE soars to $25 \mathrm{~ns}$ even with only 2 users.

In Fig.3, we show RMSE of DEMR estimator as the function of the number of users in AWGN channel. We can find that RMSE of DEMR estimator is around $0.2887 \mathrm{~ns}$ when $K$ increases from 1 to 31 , which means DEMR can achieve almost exact integer part estimation of TOA even with fullloaded system. DEMA estimator in Nyquist sampling rate can also obtain very precious TOA estimation. But in the case

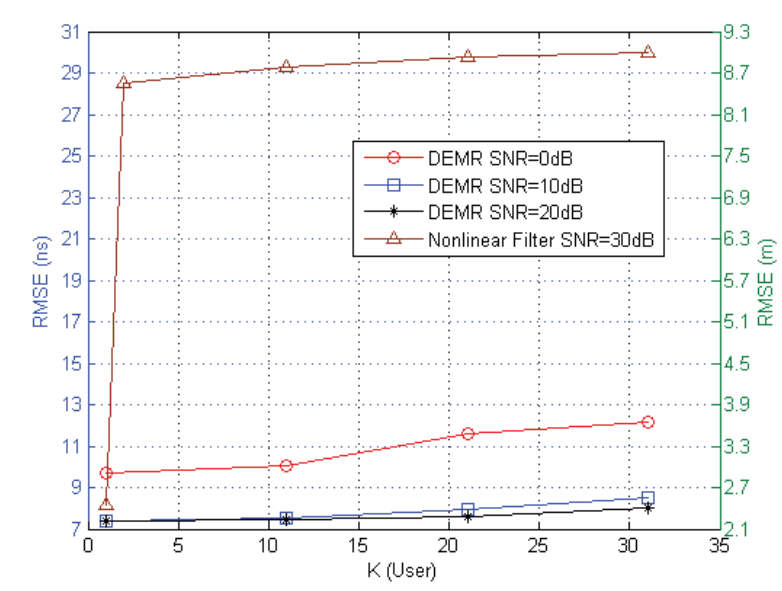

Fig. 5. RMSE versus $K$ for different SNR values with $M=100, N=31$, and near-far ratio is $10 \mathrm{~dB}$ in CM1 channel.

of chip-rate sampling, the ranging performance deteriorates to $13 \mathrm{~ns}$ even with only one user. For Nonlinear filter, RMSE increases from about $1 \mathrm{~ns}$ to $25 \mathrm{~ns}$ when $\mathrm{K}$ varies from 1 to 2 since Nonlinear filter is not efficient with $10 \mathrm{~dB}$ near-far ratio as shown in Fig.2.

Since we are more interesting in the estimator with low complexity and low sampling rate, DEMR estimator and Nonlinear filter estimator are considered in the simulation in multipath channel. In Fig.4, we investigate RMSE of DEMR and Nonlinear filter estimator with respect to near-far ratios for different SNR in CM1 channel. For DEMR estimator, RMSE increases no more than $1 \mathrm{~ns}$ when near-far ratio grows from OdB to $30 \mathrm{~dB}$ for all the SNR. However, as that in AWGN channel, Nonlinear filter cannot resist to near-far ratio even with $\mathrm{SNR}=30 \mathrm{~dB}$. The simulation results show that in the dense multipath channel, near-far problem appears to have little effect on proposed estimator.

Fig.5 shows RMSE of DEMR and Nonlinear filter estimator as a function of the number of users $K$ for different SNR in CM1 channel. For DEMR estimator, RMSE increases within 3 ns approximately as $K$ varies from 1 to 31 even with most severe noise. Especially, for the two higher SNR, the differences are less than $1 \mathrm{~ns}$. This performance is opposed to the significant degradation of Nonlinear filter estimator as $\mathrm{K}$ increases. It is shown that the DEMR estimator has capability to support larger number of users with little performance degradation.

\section{CONCLUSion}

In this paper, we have investigated the problem of multiuser ranging estimation in DS-UWB system. Low complexity DEMR estimator has been presented. Low sampling rate makes DEMR estimator very properly to low-cost ranging implementation. Compared with other low complexity TOA estimator, DEMR estimator is quite near-far resistant and can work in the fully loaded system.

\section{REFERENCES}

[1] S. Gezici, Zhi Tian, G. B. Giannakis, H. Kobayashi, A. F. Molisch, H. V. Poor, and Z. Sahinoglu, "Localization via ultra-wideband radios: 
a look at positioning aspects for future sensor networks," IEEE Signal Process. Mag., vol. 22, no. 4, pp. 70-84, Jul. 2005.

[2] D. Dardari, A. Conti, U. Ferner, A. Giorgetti, and M. Z. Win, "Ranging with ultrawide bandwidth signals in multipath environments," Proc. IEEE, vol. 97, no. 2, pp. 404-426, Feb. 2009.

[3] M. Z. Win and R. A. Scholtz, "Characterization of ultra-wide bandwidth wireless indoor channels: a communication-theoretic view," IEEE J. Sel. Areas Commun., vol. 20, no. 9, pp. 1613-1627, Dec. 2002.

[4] V. Lottici, A. D'Andrea, and U. Mengali, "Channel estimation for ultrawideband communications," IEEE J. Sel. Areas Commun., vol. 20, no. 9, pp. 1638-1645, Dec. 2002.

[5] Joon-Yong Lee and R. A. Scholtz, "Ranging in a dense multipath environment using an UWB radio link," IEEE J. Sel. Areas Commun., vol. 20 , no. 9 , pp. 1677-1683, Dec. 2002.

[6] Liuqing Yang and G. B. Giannakis, "Timing ultra-wideband signals with dirty templates,” IEEE Trans. Commun., vol. 53, no. 11, pp. 1952-1963, Nov. 2005.

[7] S. H. Song and Q. T. Zhang, "Multi-dimensional detector for UWB ranging systems in dense multipath environments," IEEE Trans. Wireless Commun., vol. 7, no. 1, pp. 175-183, Jan. 2008.

[8] I. Guvenc and Z. Sahinoglu, "Threshold-based TOA estimation for impulse radio UWB systems," in Proc. IEEE Int. Conf. Ultra-Wideband (ICUWB), Sept. 2005, pp. 420-425.

[9] Chiara Falsi, Davide Dardari, Lorenzo Mucchi, and Moe Z Win, "Time of arrival estimation for UWB localizers in realistic environments," EURASIP J. Appl. Signal Process., vol. 2006, no. 1, pp. 1-13, 2006.

[10] D. Dardari, Chia-Chin Chong, and M. Z. Win, "Threshold-based timeof-arrival estimators in UWB dense multipath channels," IEEE Trans. Commun., vol. 56, no. 8, pp. 1366-1378, Aug. 2008.

[11] E. Ekrem, M. Koca, and H. Delic, "Iterative synchronization of multiuser ultra-wideband signals," IEEE Trans. Wireless Commun., vol. 9, no. 10, pp. 3040-3051, Oct. 2010.

[12] M. Flury, R. Merz, J.-Y. Le Boudec, and J. Zory, "Performance evaluation of an IEEE 802.15.4a physical layer with energy detection and multi-user interference," in Proc. IEEE Int. Conf. Ultra-Wideband (ICUWB), Sept. 2007, pp. 663-668.

[13] Zafer Sahinoglu and Ismail Guvenc, "Multiuser interference mitigation in noncoherent UWB ranging via nonlinear filtering," EURASIP J. Wireless Commun. Netw., vol. 2006, no. 1, pp. 1-10, 2006.

[14] D. Dardari, A. Giorgetti, and M. Z. Win, "Time-of-arrival estimation of UWB signals in the presence of narrowband and wideband interference," in Proc. IEEE Int. Conf. Ultra-Wideband (ICUWB), Sept. 2007, pp. 71-76.

[15] Hongbin Li, Jian Li, and S. L. Miller, "Decoupled multiuser code-timing estimation for code-division multiple-access communication systems," IEEE Trans. Commun., vol. 49, no. 8, pp. 1425-1436, Aug. 2001.

[16] A. F. Molisch, Kannan Balakrishnan, Chia-Chin Chong, and Andrew Emami, Shahriar Fort, "IEEE 802.15.4a channel model final report," Tech. Rep., IEEE 802.15-04-0662-02-004a, 2005.

[17] A. Giorgetti and M. Chiani, "A new approach to time-of-arrival estimation based on information theoretic criteria," in Proc. IEEE Int. Conf. Ultra-Wideband (ICUWB), Sept. 2011, pp. 460-464.

[18] A. Giorgetti and M. Chiani, "Time-of-arrival estimation based on information theoretic criteria," IEEE Trans. Signal Process., 2013, doi: 10.1109/TSP.2013.2239643. 